\title{
Protein shape sampled by ion mobility mass spectrometry consistently improves protein structure prediction
}

SM Bargeen Alam Turzo ${ }^{1}$, Justin T. Seffernick ${ }^{1}$, Amber D. Rolland ${ }^{2}$, Micah T. Donor ${ }^{2}$, Sten Heinze $^{1}$, James S. Prell ${ }^{2}$, Vicki Wysocki ${ }^{1}$ and Steffen Lindert ${ }^{1, *}$

${ }^{1}$ Department of Chemistry and Biochemistry and Resource for Native Mass Spectrometry Guided Structural Biology, Ohio State University, Columbus, OH, 43210

${ }^{2}$ Department of Chemistry and Biochemistry and Materials Science Institute, University of Oregon, Eugene, OR, 97403

* Correspondence to:

Department of Chemistry and Biochemistry, Ohio State University

2114 Newman \& Wolfrom Laboratory, 100 W. $18^{\text {th }}$ Avenue, Columbus, OH 43210

614-292-8284 (office), 614-292-1685 (fax)

lindert.1@osu.edu

\begin{abstract}
$\underline{\text { Abstract }}$
Among a wide variety of mass spectrometry (MS) methodologies available for structural characterizations of proteins, ion mobility (IM) provides structural information about protein shape and size in the form of an orientationally averaged collision cross-section (CCS). While IM data have been predominantly employed for the structural assessment of protein complexes, CCS data from IM experiments have not yet been used to predict tertiary structure from sequence. Here, we are showing that IM data can significantly improve protein structure determination using the modeling suite Rosetta. The Rosetta Projection Approximation using Rough Circular Shapes (PARCS) algorithm was developed that allows for fast and accurate prediction of CCS from structure. Following successful rigorous testing for accuracy, speed, and convergence of PARCS, an integrative modelling approach was developed in Rosetta to use CCS data from IM experiments. Using this method, we predicted protein structures from sequence for a benchmark set of 23 proteins. When using IM data, the predicted structure improved or remained unchanged for all 23 proteins, compared to the predicted models in the absence of CCS data. For $15 / 23$ proteins, the RMSD (root-mean-square deviation) of the predicted model was less than $5.50 \AA$, compared to only 10/23 without IM data. We also developed a confidence metric that successfully identified near-native models in the absence of a native structure. These results demonstrate the ability of IM data in de novo structure determination.
\end{abstract}




\section{Introduction}

Proteins are at the core of virtually all cellular processes. Therefore, comprehensive knowledge of protein structures with atomistic detail can be beneficial for several pharmaceutical applications such as vaccine design ${ }^{1}$, drug discovery ${ }^{2}, 3$, enzyme design ${ }^{4}$, self-assembling molecular machines ${ }^{5}$, and many more ${ }^{6}$. Mass spectrometry (MS) has become a prominent technique in the field of structural biology due to its ability to provide structural information for proteins and protein complexes. MS can be particularly beneficial because it is faster, can work for heterogeneous samples, can be used routinely at all stages of a project, and has fewer sample preparation complications compared to high-resolution techniques such as X-ray crystallography, cryo-electron microscopy (cryo-EM) and nuclear magnetic resonance (NMR) spectroscopy. Several findings for protein structures in the gas phase also suggest that features such as elements of secondary structure, compactness and quaternary structure can be preserved during the transition from solution to desolvated state ${ }^{7-9}$. For these reasons, structural MS can be very beneficial particularly when high-resolution methods are not feasible ${ }^{10,11}$. Various methods have been developed and coupled to MS to study protein structures ${ }^{12,13}$ such as chemical crosslinking $(\mathrm{XL})^{14}$, covalent labeling $(\mathrm{CL})^{15}$, surface induced dissociation (SID) ${ }^{16}$ and other ion activation methods such as collision-induced dissociation (CID), electron capture/transfer dissociation (ExD), and ultraviolet photodissociation (UVPD), hydrogen-deuterium exchange (HDX) ${ }^{17}$ and ion mobility $(\mathrm{IM})^{18}$. While such MS techniques may provide diverse details and routine analysis of structures, experimental data collected from experiments are sparse and cannot unambiguously determine atomic-resolution structure ${ }^{19}$.

An alternative approach to experimental structure determination is to use computational modelling methods. These approaches, such as structure prediction from sequence or proteinprotein docking, can also provide insight into atomistic details of biomolecules but are frequently limited in accuracy due to the large conformational sampling space among other challenges ${ }^{20}$. While these methods can be successfully utilized in the absence of experimental data, sparse experimental data are often used to guide and improve modeling ${ }^{19,21,22}$. Experimental data from various MS techniques have recently proved pivotal within integrative structural biology frameworks ${ }^{14,17,23-40}$.

In IM, ions are transferred into an inert gas chamber at a constant pressure and temperature under the influence of a weak electric field ${ }^{41,42}$. This technique is regularly utilized to separate protein structures based on their shape and size. IM can also provide a rotationally averaged collision cross section $\left(\mathrm{CCS}_{\mathrm{IM}}\right)$ of the protein which is related to the amount of momentum exchanged between ion and buffer gas over the course of the collisions and can be thought of as somewhat like rotationally averaged cross sectional area ${ }^{43}$. Several methods have been developed to predict CCS from protein structure. Among these, the most physically realistic algorithms are the trajectory method (TJM) ${ }^{44,45}$ and diffuse trajectory method (DTM) ${ }^{46}$ which integrate Newton's equation of motion to calculate the classical scattering of gas particles. Both TJM and DTM explicitly account for long-range interactions through Lennard-Jones potentials to approximate momentum transfer from each gas particle to the collided ion. CCS obtained from these methods is extremely accurate ${ }^{45}$, but these calculations can be slow. Due to the high computational cost, prediction methods such as elastic hard sphere scattering ${ }^{47}$, projection superposition approximation (PSA) $)^{48}$, local collision probability approximation ${ }^{49}$ and projection approximation $(\mathrm{PA})^{43}$ make further approximations on TJM, resulting in faster CCS calculations. Among these approximated methods, PA is the simplest and fastest, because it neglects the scattering and longrange interactions ${ }^{43,50}$. $\mathrm{CCS}_{\mathrm{PA}}$ only accounts for the collisions of a gas particle with the ion based 
on hard sphere atomic radii by calculating the average cross-sectional area of the protein structure as experienced by the buffer gas. Using the CCS projection approximation calculation tool IMPACT, calculations are about $10^{6}$ times faster $^{43}$ than the most rigorous methods and are widely used for comparison with experimental IM data. Therefore, PA approaches are advantageous for use in integrative modeling, where the CCS calculation is required for thousands of structures that are obtained from Monte Carlo sampling.

Several instances of structural modeling in conjunction with IM data have been reported. IM spectra have been successfully predicted with the structure relaxation approximation (SRA) $\operatorname{method}^{9}$. This method uses molecular dynamics simulations to model structures in the specific

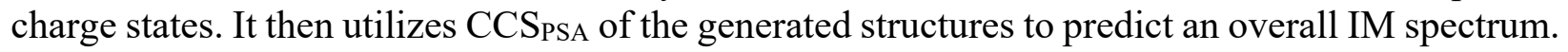
The SRA method indicated that systems studied with IM methods are generally consistent with retention of many residue-residue contacts determined by X-ray crystallography. Furthermore, IM data have been incorporated in computational modelling for protein complex structure prediction. In these methods, coarse-grained models generated using the Integrative Modelling Platform ${ }^{51}$ were ranked and clustered based on the agreement between predicted and experimental CCS measured from $\mathrm{IM}^{28,35}$. $\mathrm{CCS}_{\mathrm{IM}}$ values for complexes and their individual subunits have also been successfully used to approximate rough intersubunit distance used as restraints in modeling methods to identify coarse-grained topologies of complexes ${ }^{36-39}$. In addition to complex structure prediction, work has also been done to show correlation between IM data and structural similarity $(\text { RMSD })^{9}$. While several studies have demonstrated that IM data can be predicted and utilized with various computational methods, IM data have not yet been utilized to predict tertiary structure from sequence.

Therefore, in this work a non-stochastic grid-based algorithm, PARCS, has been implemented in Rosetta ${ }^{52,53}$ to predict CCS from structure. It has been demonstrated that PARCS yields comparable results to IMPACT in terms of speed and accuracy. Next an IM score term has been developed for use in the $a b$ initio $^{54-56}$ and comparative modelling $(\mathrm{CM})^{57}$ protocols in Rosetta, in combination with the Rosetta all-atom scoring function ${ }^{58}$. This score term scored structures based on their (dis)agreement with experimental IM data. When this score term was included, the prediction of structures improved for a benchmark of 23 proteins: the RMSD improved by an average of $2.01 \AA$ and 15/23 structures were predicted accurately.

\section{Methods}

\section{Projection approximation using rough circular shapes}

Average CCS of biomolecules are determined from IM experiments based on the amount of time required for the ion to traverse the region of inert buffer gas (usually helium or nitrogen) under the influence of a weak electric field ${ }^{43,45}$. To use IM data in a structure prediction protocol, we developed Projection Approximation using Rough Circular Shapes (PARCS) in Rosetta. The schematic (A) and the illustration (B) in Figure 1 demonstrate how the PARCS algorithm computes CCS from structure and estimates area of a projection, respectively. The PARCS algorithm, as shown in Figure 1A, takes 3D atomic protein coordinates as input. Next, the structure is randomly rotated. For each rotation, the structure is projected on a 2D grid (grid cell area of $1 \AA^{2}$ ) in the $x$ $y, x-z$, and $y-z$ planes as shown in Figure 1A (i). In the 2D grid, the projection of the protein is centered, and the grid extends $5 \AA$ beyond the most extreme atom in each direction. For each atom on the 2D grid (Figure 1A(ii)), the center grid cell is filled as denoted by the blue grid cell in Figure 1B (ii). Then, eight additional cells (red grid cells in Figure 1B (ii)) are also filled. The distance of these eight grid cells from the central cell (i.e., radius of the circular projection) is based on the 
sum of the radii of the projected atom and the buffer gas ( $r$ in Figure 1B (ii)). An effective atomic cross-sectional radius of $1.91 \AA$ is used for heavy atoms (carbon, sulfur, oxygen, nitrogen and phosphorous) and 1.21 $\AA$ is used for hydrogen atoms. A buffer gas radius of $1.0 \AA$ and $1.82 \AA$ is used in the case of helium ${ }^{43}$ and nitrogen $^{59}$,

respectively. The eight points are positioned such that two adjacent points on the circumference form a $45^{\circ}$ angle from the center point as shown in Figure 1B (ii). This process is repeated for all atoms in the protein, filling the overall grid as shown in Figure 1B (iii). Finally, the projection area $(A)$ is derived by summing the areas of the filled

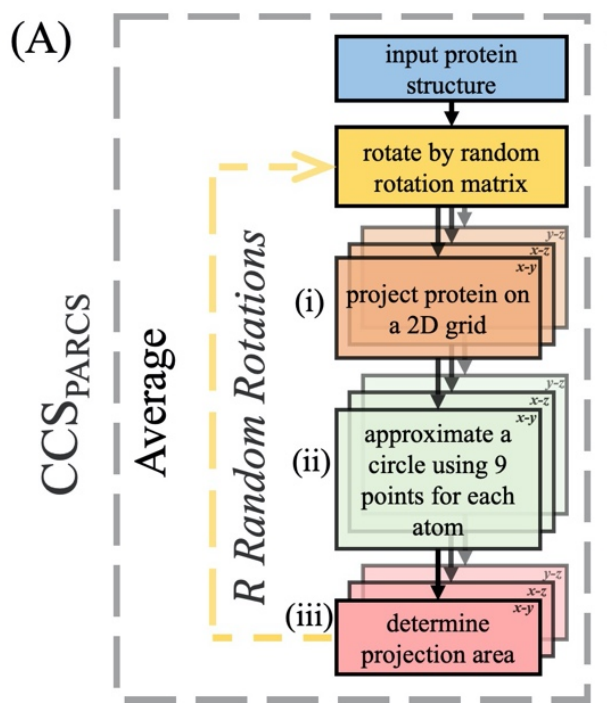

(B)

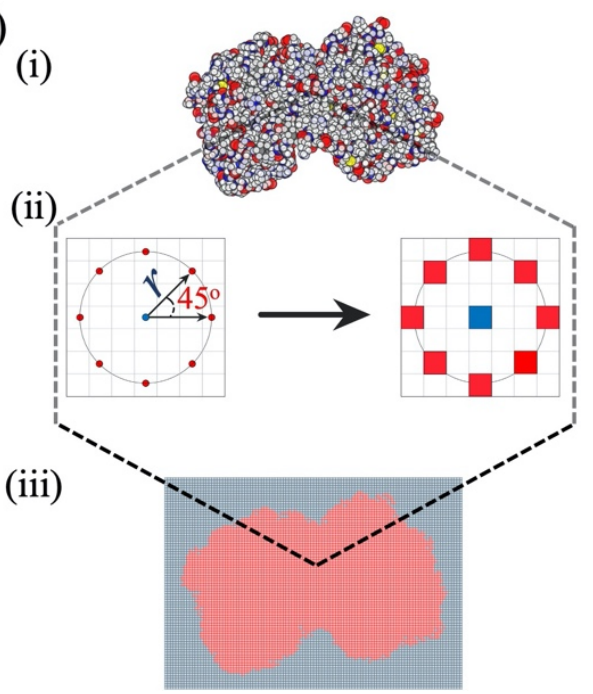

Figure 1: (A) Schematic of the PARCS algorithm to predict CCS from structure. (i) Three projections are obtained from each rotation. (ii) For each atom in each projection the 2D grid is filled according to a 9-point circle approximation. (iii) The projection area is determined from the number of filled grid cells. (B) (i) Illustration of a 2D projection of a single random rotation where the carbon, sulfur, oxygen, nitrogen, and hydrogen are colored grey, yellow, red, blue, and white respectively. (ii) Each atom is projected on a grid with a cell size of $1 \AA^{2}$. The center grid cell and eight other grid cells at a distance $r$ (based on the radii of the given atom and the buffer gas) from the center of the atom are filled. (iii) Projection of the randomly rotated protein after the grid cells are filled according to the PARCS algorithm. grid cells. From the $x-y$, $y-z$, and $x-z$ projections for each random rotation $i$, three projection areas $\left(A_{i}^{x-y}, A_{i}^{x-z}\right.$ and $\left.A_{i}^{y-z}\right)$ are obtained. The CCS of the structure $\left(C C S_{P A R C S}\right)$ is then acquired from the average area of the total number of projections ( $\mathrm{N}=3 \mathrm{R}$, where $R$ is the total number of random rotations) as shown in Equation 1.

$$
\operatorname{CCS}_{P A R C S}=\frac{\sum_{i}^{R}\left(A_{i}^{x-y}+A_{i}^{x-z}+A_{i}^{y-z}\right)}{N}
$$

\section{IM score function in Rosetta}

CCS from experimental IM data were incorporated as a spatial restraint for integrative Rosetta modelling as it provides information about protein size and shape. Therefore, to integrate this information in Rosetta for protein structure prediction, a score term (IM Score) was developed to quantify agreement of protein structures with IM data, using CCS as the restraint. The evaluation score, $E_{I M}$, was defined as a sum of the $I M_{\text {Score }}$ score term with the Rosetta REF2015 score function $^{58}$ as shown in Equation 2. 


$$
E_{I M}=E_{\text {Rosetta }}+I M_{\text {Score }}
$$

In Equation 2, $E_{\text {Rosetta }}$ is the energy of the structure obtained from the Rosetta REF2015 score function. The IMscore term is a penalty function (as defined and shown in Equation 3 and Figure S1, respectively) based on the absolute difference ( $\triangle \mathrm{CCS}$ ) between $\mathrm{CCS}_{\text {PARCS }}$ and $\mathrm{CCS}_{\mathrm{IM}}$. This function includes a lower bound (LB) and an upper bound (UB) cutoff (as shown in Equation $3)$ to account for error ${ }^{24}$. $\triangle$ CCS below LB $\left(10 \AA^{2}\right)$ are not penalized and $\triangle C C S$ above UB $\left(100 \AA^{2}\right)$ are given a maximum penalty of 100 , with a fade function used in between. Conceptually, this scoring function penalizes structures with high deviation from experiment.

$$
\begin{gathered}
\mathrm{IM}_{\text {Score }}=\left\{\begin{array}{cl}
0 & \text { if } \triangle C C S<L B \\
100\left(2 x^{3}-3 x^{2}+1\right) & \text { if } L B<\Delta C C S<U B \\
100 & \text { if } \triangle C C S>U B \\
x=-\left(\frac{\Delta C C S-U B}{U B-L B}\right)
\end{array}\right.
\end{gathered}
$$

\section{IM datasets}

In this work, our aim was to study predominantly globular and ordered proteins within all

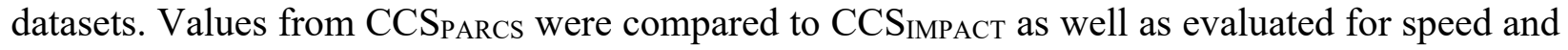
precision on 4465 non-homologous protein structures (PARCS evaluation dataset) extracted from the protein databank (PDB $)^{60}$ (http://www.rcsb.org/) using the PISCES ${ }^{61}$ webserver (http://dunbrack.fccc.edu/pisces). For this dataset, the sequence identity was less than or equal to $10 \%$, sequence length was between $40-250$ residues, non-X-ray and CA-only entries were excluded and the PDBs were culled by chain. For CCS prediction and speed comparison, PARCS was benchmarked against IMPACT ${ }^{43}$ (with flag ' $-\mathrm{H}$ ' to include hydrogens) based on the calculations performed on the PARCS evaluation dataset. This dataset was also used to test the convergence of PARCS with respect to the number of rotations. In this convergence test, the standard deviation of 100 separate CCS calculations for each protein at varying numbers of rotations were obtained and assessed for the optimal number of random rotations required for calculations to converge.

To evaluate the ability of the score term (Equation 3 ) to distinguish native from non-native protein models in the case of an error-free CCS prediction, a set of 100 proteins was randomly selected from the PDB (list of monomers shown in Table S1) using the PISCES webserver, where the sequence length ranged from 24 to 154. A set of structure prediction experiments (which will be described in detail in the following sections) was performed on this dataset, where the experimental CCS was simulated by predicting CCS of the native structure with PARCS. Therefore, this dataset was referred to as the ideal dataset. The simulated CCS values ranged from $595 \AA^{2}$ to $1710 \AA^{2}$ for the 100 proteins in the ideal dataset. The score function was also tested on actual experimental IM data, i.e., structures with $\mathrm{CCS}_{\mathrm{IM}}$ (experimental dataset). The experimental dataset $^{18,62-66}$ consisted of 23 monomeric proteins that also had structural information deposited in the PDB (as outlined in Table S2). Sequence lengths ranged from 26-691 residues and CCS IM 
values (for the lowest charge states) ranged from $588 \AA^{2}$ to $4580 \AA^{2}$. Additionally, these proteins exhibited an average percent disorder of only $13 \%$ as calculated by the Rosetta ResidueDisorder ${ }^{67}$, 68 application.

\section{$A b$ initio and comparative modeling protocol for structure prediction}

To test whether shape and size information encoded in IM data were sufficient to discriminate between low and high RMSD models of single-subunit proteins, we tested our algorithm on both the ideal and experimental dataset. For these two datasets, the Rosetta ab initio protocol was used for proteins with sequence length less than 155 residues, otherwise the Rosetta multi-template comparative modeling (CM) protocol was used. The templates and weights associated with all proteins for CM are provided in Table S4. The 3mers and 9mers fragments required for both protocols were generated using the fragment picker tool ${ }^{69}$ in Rosetta. The protocols ( $a b$ initio and $\mathrm{CM}$ ) for both the ideal and experimental data set are further detailed in the SI. All structures generated from the $a b$ initio and comparative modeling protocols were subjected to the Rosetta Relax protocol. The IM data, ideal and experimental, were then used to score all the structures generated for each protein in Table S1 and Table S2, respectively. The top scoring model was designated as the predicted structure.

\section{Analysis metrics used for evaluating predictions}

We quantitatively assessed the quality of our predicted models using several of the following metrics. The global RMSDs (root-mean-square deviations) of the predicted models to their native structures were calculated. Predictions with IM data where RMSD was within $0.5 \AA$ of the RMSD of the structure predicted without IM data were defined as unchanged. Next, $\mathrm{P}_{\text {near }}{ }^{70}$, a goodness-of-energy funnel metric (at $k_{B} T$ and $\lambda$ set to 4 and $3 \AA$ respectively), was used to compare the score versus RMSD distributions predicted with and without IM data. $\mathrm{P}_{\text {near }}$ ranges from 0 (a poor energy funnel) to 1 (a well-defined energy funnel). Predicted structures from both ideal and experimental datasets were evaluated with these two metrics. The predicted structures from the experimental dataset were further evaluated with the template modeling score (TMscore) ${ }^{71}$ and global distance test total score (GDT_TS $)^{72}$. Template modeling score (TM-score) was used to assess the topological similarity of the predicted structures to native structures using the TM-score program ${ }^{71}$. The TM-score metric ranges from 0 to 1 , where scores below 0.17 indicate randomly chosen unrelated proteins and a score higher than 0.5 corresponds to structures being generally in the same fold and a score of 1 indicates a perfect match. Global distance test total score (GDT_TS) values were used to assess the optimal superposition between the predicted and the native structure by identifying groups of residues in the predicted model that differ from that of the native structure by less than a distance cutoff. GDT_TS for the C $\alpha$ atoms at a distance cutoff of $5 \AA$ was calculated using the LGA program ${ }^{72}$. GDT_TS values range from 0 to 100 where higher GDT_TS is indicative of a predicted structure being similar to the native structure.

\section{Confidence metric used for identifying accurate and inaccurate predictions}

A metric was developed to quantify confidence in predictions in the absence of known structure. This confidence metric was defined as the average per-residue score of the top 100 scoring models predicted with IM data (using Equation 2). The specific metric was chosen because lower scores per residue are generally associated with more native-like structures. Thus, structures were defined as high confidence if the average residue score was less than -2.54 (above which structures were defined as low confidence). Instances where the RMSD of the prediction was less 
$5.50 \AA$ and average residue score less than -2.54 were considered successful confidence measure cases. We chose an RMSD cutoff of $5.5 \AA$ since below that RMSD, protein topologies are generally predicted correctly.

\section{$\underline{\text { Results and discussion }}$}

In this study, to utilize IM data to predict tertiary (monomeric) structures in Rosetta, an algorithm designed for rapid prediction of CCS has been developed and implemented. This method uses Projection Approximation via a grid-based calculation of Rough Circular Shapes (PARCS). Subsequently, a score function was developed that assessed the agreement of Rosetta-generated models to the $\mathrm{CCS}_{\mathrm{IM}}$ for tertiary structure prediction.

\section{Calculation of CCS using PARCS was fast, accurate, and results were comparable to existing software} Area

calculation in projection approximation methods is typically performed using Monte Carlo integration methods. In such an approach, probes representing the buffer gas particle are fired upon the randomly oriented 2Dprojected target structure to calculate the area of the projection. A large number of probes is usually required for CCS calculations to converge. However, when a large number of probes is used,
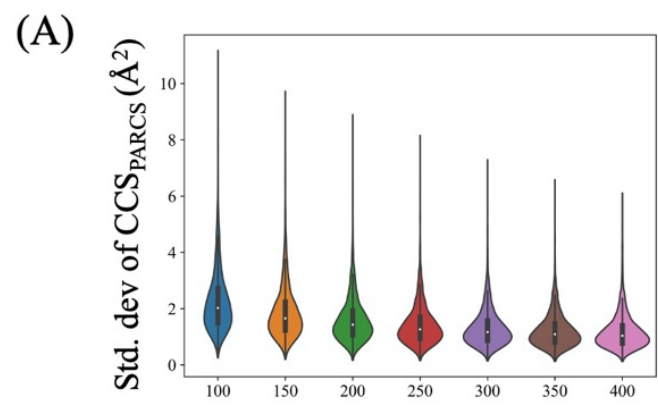

(C)

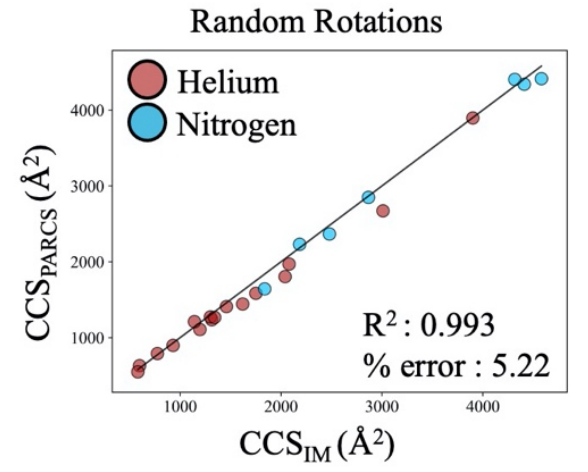

(B)

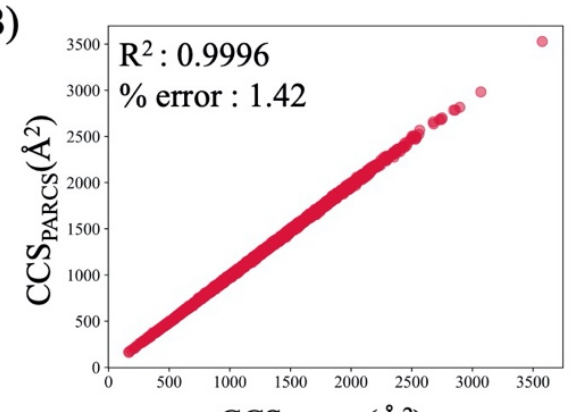

(D)

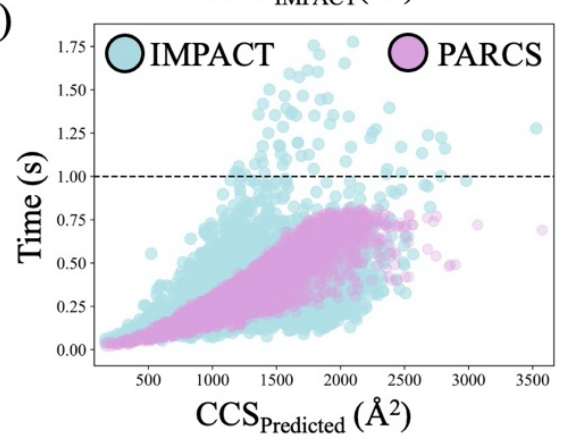

Figure 2: Analysis of PARCS algorithm. (A) Convergence of PARCS calculation over 100-400 random rotations. For more than 250 rotations the mean standard deviation was below $1.5 \AA^{2}$ (B) Comparison of CCS PARCs to that of $\mathrm{CCS}_{\text {IMPACT }}$ exhibited a very strong correlation $\left(\mathrm{R}^{2}\right.$ of 0.9996$)$. (C) A strong correlation $\left(\mathrm{R}^{2}\right.$ of 0.993$)$ was observed for predicted CCSPARCS values of PARCS when compared with $\mathrm{CCS}_{\mathrm{IM}}$ from nitrogen (blue) and helium (red) buffer gas for the experimental dataset. (D) Comparison of CCS calculation time of PARCS and IMPACT showed that PARCS and IMPACT performed equally well in terms of speed.

random probes frequently survey areas with no protein present, resulting in unnecessary calculations and thus adding to the computational $\operatorname{cost}^{73}$. Therefore run-to-run variability in probebased projection area calculation per rotation is common. To circumvent this issue, in PARCS, the projection area is calculated by projecting the structure on a $2 \mathrm{D}$ grid and then geometrically estimating the projection area directly (by geometrically filling the grid based on locations of 
atoms and radii of atoms and probes). This approach eliminates the variability in projection area calculation. Therefore, the only attribute contributing to the variability in CCS calculations using PARCS is the random rotations.

To benchmark our PARCS algorithm, CCS values for 4465 non-homologous protein structures in the PARCS evaluation dataset were calculated. Results for convergence of CCS calculations at varying number of random rotations on the PARCS evaluation dataset are shown in Figure 2A. The average standard deviation of the CCS distributions for 100 rotations was only $2.26 \AA^{2}$ (which was less than $0.2 \%$ of the CCSPARCS on average) and decreased as the number of rotations increased. The average of the standard deviations of the CCS distributions was well below $2.0 \AA^{2}$ for more than 100 rotations as shown in Figure 2A and summarized in Table 1. For CCSPARCS, the default number of rotations was set to 300, where the average standard deviation of the distribution was $1.31 \AA^{2}$.

Table 1: Average standard deviation of CCS calculations for proteins in the PARCS evaluation dataset at various rotations.

\begin{tabular}{|c|c|}
\hline Rotations & Avg std dev $\left(\AA^{2}\right)$ \\
\hline 100 & 2.26 \\
150 & 1.85 \\
200 & 1.60 \\
250 & 1.42 \\
300 & 1.31 \\
350 & 1.22 \\
400 & 1.16 \\
\hline
\end{tabular}

For all proteins in the PARCS evaluation dataset, CCS calculated by PARCS was compared to CCS calculated by IMPACT, one of the most widely used CCS calculation methods, as shown in Figure 2B. A strong correlation $\left(\mathrm{R}^{2}=0.9996\right)$ was observed between CCS CCS IMPACT with a root mean squared error (RMSE) of $20.38 \AA^{2}$ and an average percent error of $1.42 \%$. The results demonstrate that PARCS calculates CCS values as accurately as other projection approximation methods. CCS PARCS were then compared to $\mathrm{CCS}_{\text {IM }}$ for the experimental dataset. We observed a strong correlation $\left(\mathrm{R}^{2}\right.$ PARCS $\left.=0.993\right)$ between $\mathrm{CCS}_{\text {PARCS }}$ and $\mathrm{CCS}_{\text {IM values }}$ as shown in Figure 2C, where IM data collected in nitrogen and helium buffer gas are shown in blue and red respectively. We observed an average percent error of only $5.22 \%$ (similar to that of IMPACT at 5.61\%) from $\mathrm{CCS}_{\mathrm{IM}}$. To use IM data in computational structure prediction methods (where CCS prediction is required on a large number of decoy structures), the speed of CCS calculations should be within about a second. Therefore, calculation times of PARCS were compared to that of IMPACT as presented in Figure 2D. Using the PARCS evaluation dataset, PARCS took an average of 0.40 seconds to calculate the CCS of proteins when 300 random rotations were used compared to 0.32 seconds for IMPACT. Thus, the timing of PARCS was comparable to IMPACT. We note that the slightly longer average time for PARCS was due to additional steps performed by Rosetta when reading in a PDB file (such as checks for correctness and adding missing hydrogens ${ }^{52}$ ). For all 4465 proteins, calculations for PARCS completed in under 1.0 second as shown in Figure 2D. These results indicate that PARCS in Rosetta offers 
similar speed and accuracy to established PA algorithms. We hypothesized that the information contained within $\mathrm{CCS}_{\mathrm{IM}}$ may be sufficient to predict structures using CCS PARCs.

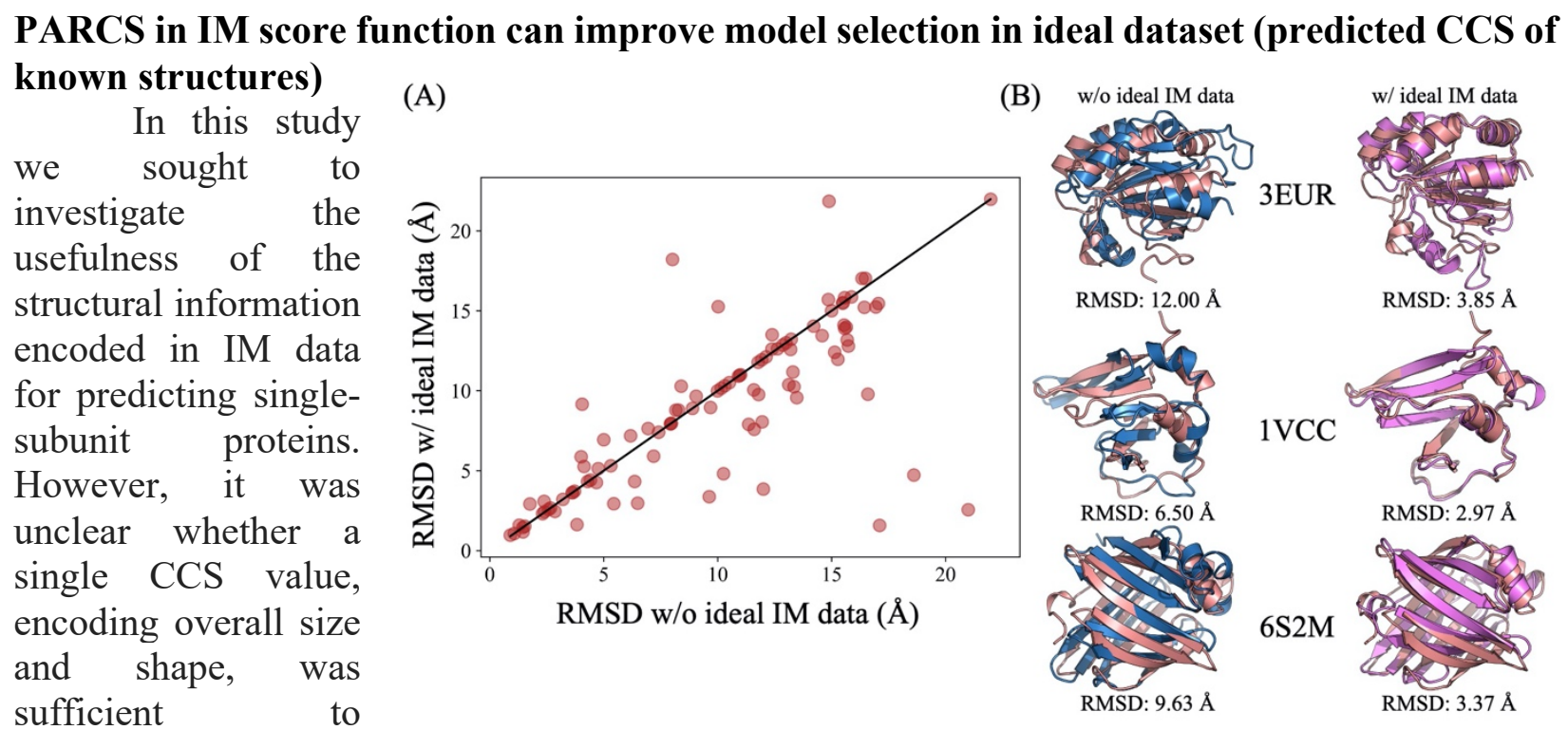

distinguish nearnative from incorrect protein models. To test how useful the information in CCS was for structure

Figure 3: (A) Comparison of the RMSD of the predicted structure of 100 proteins in the ideal dataset. The RMSD improved or remained unchanged for 82 proteins when ideal IM data were used for scoring. (B) Comparison of predicted structures without (blue) and with (pink) the inclusion of ideal IM data to their native structures (salmon).

prediction, an IM score function has been developed to score structures based on the (dis)agreement with experimental IM data. To assess the capabilities of this score function to adequately distinguish good from bad models, we first tested it on the ideal dataset (where the experimental $\mathrm{CCS}_{\text {IM }}$ was replaced with CCS PARCS of the native structure). For each protein in the ideal dataset 10,000 potential structures were generated using the $a b$ initio protocol and scored using the developed IM score function $\left(\mathrm{E}_{\mathrm{IM}}\right)$. Prediction results with and without the inclusion of ideal IM data were evaluated and compared based on agreement with experimental structures (using the RMSD of the lowest scoring model, i.e., the predicted structure). We observed a significant improvement in model quality upon the inclusion of ideal IM data. As highlighted in Figure 3A, the RMSD of the predicted structures was improved or unchanged for 82 out of 100 proteins when ideal IM data were included in the scoring. Three of these improvements are shown in Figure 3B, where the native structure (salmon) was compared to the predicted model without and with the inclusion of ideal IM data in blue and pink, respectively.

For the subset of models where the RMSD improved, it improved by an average of $3.62 \AA$. While the results also suggest that 18 proteins showed an undesirable increase in RMSD (when compared to selection with $E_{\text {Rosetta }}$ ), the average increase in RMSD of this subset was only 2.35 $\AA$. Within these 18 proteins, there were only four cases where the RMSD increased by more than $2 \AA$. However, three of those four cases were not correctly predicted without the aid of ideal IM data either. The overall average RMSD improved by $0.93 \AA$ for the ideal dataset. Along with the improvement of model selection, this score function also improved definition of the energy funnel, as quantified by the $\mathrm{P}_{\text {near }}$ metric. From the $\mathrm{P}_{\text {near }}$ analysis we saw a 7.3-fold increase upon the 
inclusion of ideal IM data. This suggests that inclusion of IM data significantly improved the goodness of the score versus RMSD funnel. These results indicated that the overall size and shape information contained in the IM data indeed had a strong potential to facilitate the discrimination of good from bad models. Given the sparseness of the data (CCS is a single number denoting the average cross-sectional area of the protein) the improvement was quite significant. While an encouraging proof of principle, these results did not account for the uncertainty associated with real experimental IM data. When experimental IM data are used for the structure prediction, additional uncertainty is included (an average percent error of $5.22 \%$ between CCSPARCS and $\mathrm{CCS}_{\text {IM }}$ was observed for the experimental dataset). Therefore, the effectiveness of IM data to improve structure prediction still needed to be tested on a dataset with experimental IM data.

\section{IM data improved model selection of protein structures in experimental dataset}

For

proteins in the

experimental

dataset, $\quad 10,000$

decoy models

were generated

with the $a b$ initio

protocol for

proteins with

fewer than 155

residues and

comparative

modeling (CM)

for proteins with

more than 155

residues. Each of

these decoy

models was then

scored with IM

data (Equation 2)

and the predicted

models without

and with data
(A)

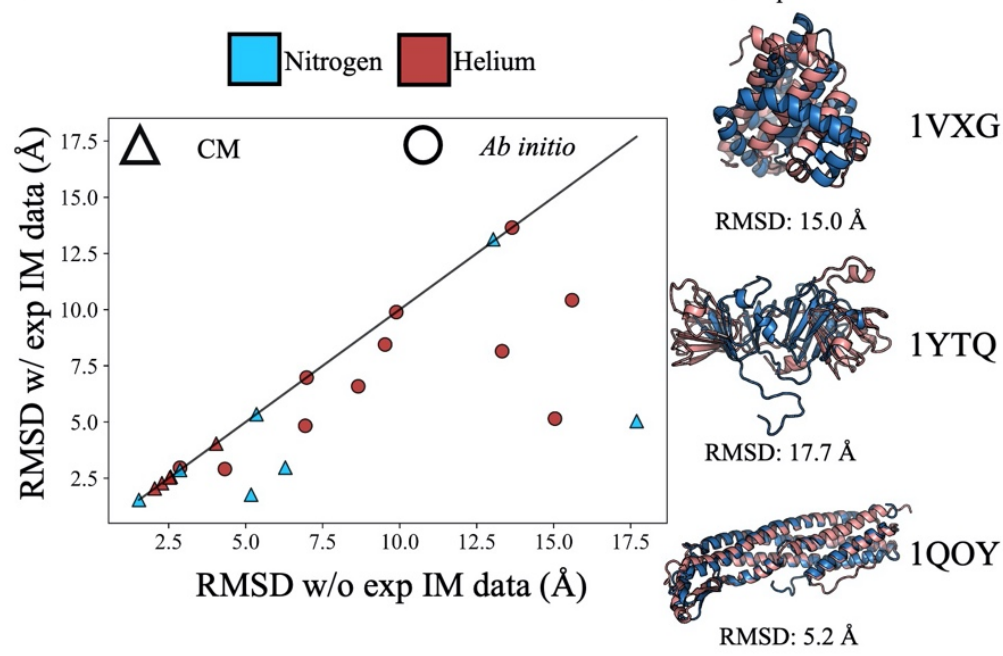

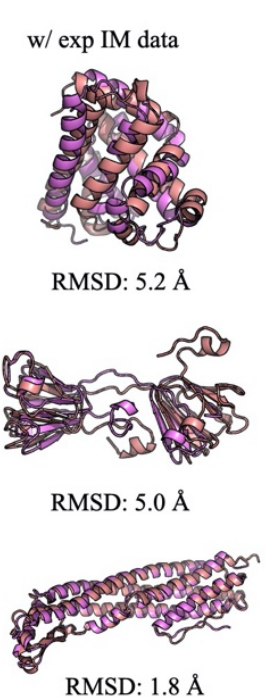

RMSD: $1.8 \AA$

Figure 4: (A) RMSD of the best scoring models with IM data (blue and red for nitrogen and helium buffer gas respectively) when compared to RMSD without IM data for 23 proteins in the experimental dataset. Structures predicted with $\mathrm{CM}$ and $a b$ initio are represented with a triangle and circle respectively. (B) Comparison of predicted structures without (blue) and with (pink) the inclusion of IM data to their native structures (salmon).

were compared. Again, we saw a notable improvement in model quality upon the inclusion of IM data. In Figure 4A, the RMSDs of the best scoring models without and with IM data are shown. The RMSD of the predicted models for proteins in the experimental dataset either improved or remained unchanged in all 23 cases. The RMSD improved by an average of $2.01 \AA$ when IM data were utilized as restraints. Of these 23 cases, 15 proteins were ultimately predicted with an RMSD of less than $5.50 \AA$, compared to 10 proteins without IM data. Furthermore, it was observed that for the subset of proteins where the CM and $a b$ initio protocols (shown as triangles and circles respectively in Figure 4A) were used for model generation, the average RMSD improved by 1.61 $\AA$ and $2.44 \AA$ respectively. Figure 4B shows structures of the predicted models (aligned to the native models in salmon) obtained without and with the inclusion of IM data (in blue and pink, respectively). The largest improvement was observed for the system $\beta$-crystallin B2 (PDB ID: 
1YTQ), which improved from $17.7 \AA$ to $5.0 \AA$. The score vs RMSD distributions for several benchmark proteins before (blue) and after (pink) scoring with IM data are shown in Figure 5. In these distributions, the predicted models without and with IM data are marked with a blue and pink star, respectively. We also observed a general improvement in $P_{\text {near }}$ upon scoring with IM data with a 4.5-fold average improvement.

Additional analyses on the predicted structures of the experimental dataset further revealed general improvements upon the inclusion of IM data. TM score analysis of this dataset suggested improvements with IM data. We observed, as shown in Table S5, that $74 \%$ of the proteins had a TM-score greater than (or equal to) 0.5 when IM data were incorporated as

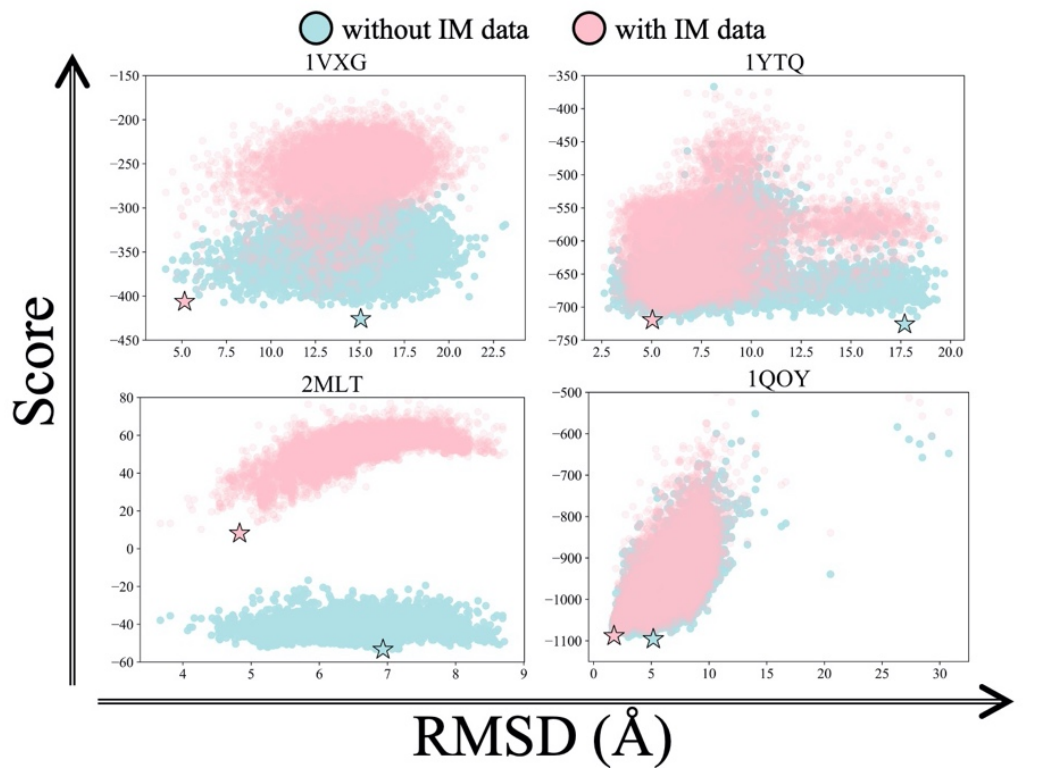

Figure 5: Score vs RMSD plots without (blue) and with (pink) IM data for four proteins that showed significant improvement in the experimental dataset. The best scoring models from both predictions are marked with a blue and pink star, respectively. opposed to $65 \%$ without the use of IM data. The TM-scores of the best scoring models of the CM subset were generally higher than those of the ab initio subset, while the biggest improvements were observed for the $a b$ initio subset. General improvements were also seen in a GDT_TS analysis of the best models predicted upon the inclusion of IM data with an average percent score improvement of $2.88 \%$. Similarly, the average GDT_TS of the CM protein subset was higher than that of the ab initio subset. In all cases, improvements were observed when IM data were used, with average improvements of $1.51 \%$ and $4.39 \%$ for the CM and ab initio subsets, respectively. All results, as summarized in Table 2, demonstrated that experimental IM data can offer useful information regarding the structural shape of the protein which consequently aids in improved scoring and model selection.

Table 2: Metric analysis for the best scoring model with and without IM data for the experimental dataset.

\begin{tabular}{|c|c|c|c|c|}
\cline { 2 - 5 } \multicolumn{1}{c|}{} & $\begin{array}{c}\text { Avg } \\
\text { RMSD } \\
(\AA)\end{array}$ & $\begin{array}{c}\text { Avg } \\
\text { GDT_TS } \\
(\mathbf{\%})\end{array}$ & $\begin{array}{c}\text { Avg } \\
\text { TM- } \\
\text { Tcore }\end{array}$ & $\begin{array}{c}\text { Avg P Pear } \\
\text { ratio }\end{array}$ \\
\hline w/o IM data & 7.49 & 58.35 & 0.63 & 1.00 \\
\hline CM & 5.45 & 69.41 & 0.82 & 1.00 \\
ab initio & 9.71 & 46.29 & 0.43 & 1.00 \\
\hline w/ IM data & 5.48 & 61.23 & 0.66 & 4.54 \\
\cline { 1 - 4 }
\end{tabular}




\begin{tabular}{|c|c|c|c|c|} 
& 3.84 & 70.92 & 0.84 & 1.74 \\
ab initio & 7.27 & 50.68 & 0.47 & 8.18 \\
\hline
\end{tabular}

\section{Confidence measure successfully discriminated accurate and inaccurate models}

The inclusion of

IM data helped improve structure prediction for all 23 proteins in the experimental dataset. However, there were 8 cases where the RMSD of the selected model (even after improvement) was greater than $5.5 \AA$. This knowledge was available to us since the native structures were known for the models generated within this benchmark dataset. However, in true blind structure prediction protocols, RMSD information is not available. For this reason, we developed a confidence measure that allowed us to selectively flag successful

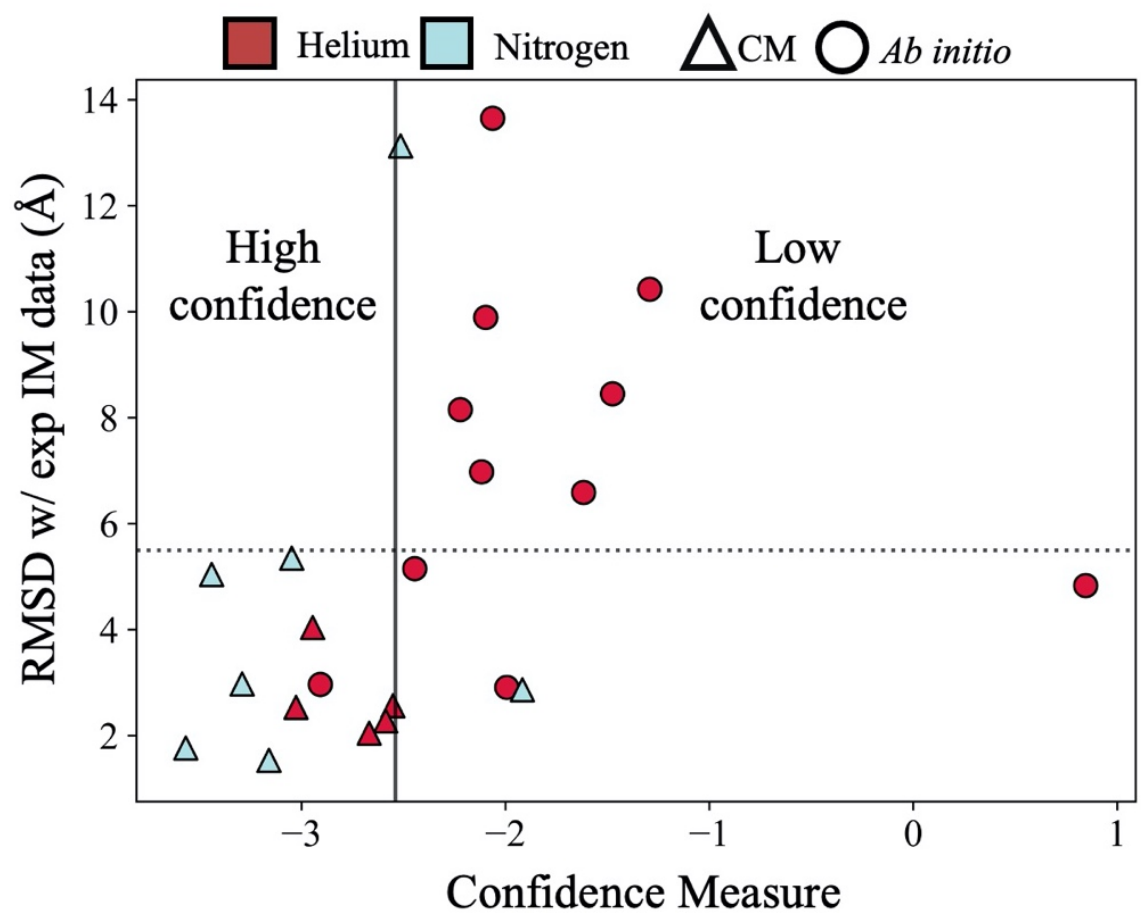
prediction cases in the

Figure 6: Confidence measure (defined as average residue score of top 100 predicted models) successfully separated the best scoring models from the experimental dataset into two groups, high confidence, and low confidence.

absence of native structure. The confidence measure was defined as the average residue score of the top 100 scoring models. According to this metric analysis, the high and low confidence structures were separated by a score cutoff of -2.54 . This metric successfully flagged all inaccurate predictions as low confidence, whereas all high confidence predictions were accurate as shown in Figure 6. We saw similar trends when we used this confidence metric to evaluate top scoring models from the ideal dataset as shown in Figure S2.

\section{Conclusion}

Ion mobility has emerged as a prime tool to study proteins in their native states using MS. However, the information obtained is sparse, not directly allowing for full structure elucidation. Thus, computational techniques are needed to deduce structural information from IM data. In this study we developed the first such algorithm for structure prediction of single subunit proteins from IM data. To achieve this, we first developed a method (PARCS) that could predict CCS from structures, which has been implemented in Rosetta as a stand-alone application. Following the successful benchmarking of this application, a score term, based on restraints derived from IM data, has been developed to predict native-like structures. This score term was tested on a set of 
100 structures from the PDB, where CCSPARCS of the native structure was treated as the experimental $\mathrm{CCS}_{\mathrm{IM}}$. This was done, as a proof of principle, to check if the score function could translate the structural information (encoded in IM data) into spatial restraints in the absence of model error. Based on RMSD analysis, we observed that the inclusion of IM data improved structure prediction results for 82 out of 100 structures. Following this positive validation, the score function was tested on a benchmark set of 23 proteins with experimental IM data. We showed that IM data improved model selection, as demonstrated by analyzing the best scoring models with several metrics. We also developed a confidence metric to successfully separate good predictions from bad predictions in the absence of native structure. Our current computational workflow illustrates that CCS obtained from IM experiments, despite its sparseness, provides sufficient information on the overall shape and size of proteins to be used as restraints to improve model selection in protein structure prediction. This study also further demonstrates the close connection between the solution and gas phase structure in native IM techniques. Our developed CCS calculation method and score function are freely and easily accessible through Rosetta Commons. A tutorial with examples on how to use the PARCS application as well as the use of IM data in structure prediction has been included in the SI. Further work will focus on improving methods to incorporate CCS data for protein complexes using RosettaDock ${ }^{74}$ and on the use of multiple complementary MS data (such as the combination of CL and/or SID data with IM data) for protein and complex structure prediction in Rosetta.

\section{Acknowledgments}

We thank the members of the Lindert lab for many useful discussions. We would like to thank the Ohio Supercomputer Center for valuable computational resources ${ }^{75}$. We also thank Alyssa Stiving and Benjamin Jones for the data they collected on beta lactoglobulin, carbonic anhydrase, ubiquitin and serum albumin. This work was supported by NIH (P41 GM128577, R21A125804). Additionally, integrative protein modeling was supported by a Sloan Research Fellowship to S.L. 


\section{References}

1. Huang, P.-S.; Boyken, S. E.; Baker, D., The coming of age of de novo protein design. Nature 2016, 537 (7620), 320-327.

2. Coelho, E. D.; Arrais, J. P.; Oliveira, J. L., From protein-protein interactions to rational drug design: are computational methods up to the challenge? Curr Top Med Chem 2013, 13 (5), 602-18.

3. Leelananda, S. P.; Lindert, S., Computational methods in drug discovery. Beilstein J Org Chem 2016, 12, 2694-2718.

4. Kiss, G.; Çelebi-Ölçüm, N.; Moretti, R.; Baker, D.; Houk, K. N., Computational enzyme design. Angew Chem Int Ed Engl 2013, 52 (22), 5700-25.

5. $\quad$ King, N. P.; Sheffler, W.; Sawaya, M. R.; Vollmar, B. S.; Sumida, J. P.; André, I.; Gonen, T.; Yeates, T. O.; Baker, D., Computational design of self-assembling protein nanomaterials with atomic level accuracy. Science 2012, 336 (6085), 1171-4.

6. Khoury, G. A.; Smadbeck, J.; Kieslich, C. A.; Floudas, C. A., Protein folding and de novo protein design for biotechnological applications. Trends Biotechnol 2014, 32 (2), 99-109.

7. Wyttenbach, T.; Bowers, M. T., Structural stability from solution to the gas phase: native solution structure of ubiquitin survives analysis in a solvent-free ion mobility-mass spectrometry environment. J Phys Chem B 2011, 115 (42), 12266-75.

8. Ruotolo, B. T.; Robinson, C. V., Aspects of native proteins are retained in vacuum. Curr Opin Chem Biol 2006, 10 (5), 402-8.

9. Bleiholder, C.; Liu, F. C., Structure Relaxation Approximation (SRA) for Elucidation of Protein Structures from Ion Mobility Measurements. J Phys Chem B 2019, 123 (13), 2756-2769.

10. Artigues, A.; Nadeau, O. W.; Rimmer, M. A.; Villar, M. T.; Du, X.; Fenton, A. W.; Carlson, G. M., Protein Structural Analysis via Mass Spectrometry-Based Proteomics. Advances in experimental medicine and biology 2016, 919, 397-431.

11. Danev, R.; Yanagisawa, H.; Kikkawa, M., Cryo-Electron Microscopy Methodology: Current Aspects and Future Directions. Trends Biochem Sci 2019, 44 (10), 837-848.

12. Leney, A. C.; Heck, A. J. R., Native Mass Spectrometry: What is in the Name? Journal of the American Society for Mass Spectrometry 2017, 28 (1), 5-13.

13. Allison, T. M.; Barran, P.; Cianférani, S.; Degiacomi, M. T.; Gabelica, V.; Grandori, R.; Marklund, E. G.; Menneteau, T.; Migas, L. G.; Politis, A.; Sharon, M.; Sobott, F.; Thalassinos, K.; Benesch, J. L. P., Computational Strategies and Challenges for Using Native Ion Mobility Mass Spectrometry in Biophysics and Structural Biology. Anal Chem 2020, 92 (16), 10872-10880.

14. Matthew Allen Bullock, J.; Schwab, J.; Thalassinos, K.; Topf, M., The Importance of Non-accessible Crosslinks and Solvent Accessible Surface Distance in Modeling Proteins with Restraints From Crosslinking Mass Spectrometry. Mol Cell Proteomics 2016, 15 (7), 2491-500.

15. Mendoza, V. L.; Vachet, R. W., Probing protein structure by amino acid-specific covalent labeling and mass spectrometry. Mass Spectrometry Reviews 2009, 28 (5), 785-815.

16. Zhou, M.; Wysocki, V. H., Surface induced dissociation: dissecting noncovalent protein complexes in the gas phase. Acc Chem Res 2014, 47 (4), 1010-8.

17. Roberts, V. A.; Pique, M. E.; Hsu, S.; Li, S., Combining H/D Exchange Mass Spectrometry and Computational Docking To Derive the Structure of Protein-Protein Complexes. Biochemistry 2017, 56 (48), 6329-6342. 
18. Jurneczko, E.; Barran, P. E., How useful is ion mobility mass spectrometry for structural biology? The relationship between protein crystal structures and their collision cross sections in the gas phase. Analyst 2011, 136 (1), 20-28.

19. Seffernick, J. T.; Lindert, S., Hybrid methods for combined experimental and computational determination of protein structure. J Chem Phys 2020, 153 (24), 240901.

20. Kuhlman, B.; Bradley, P., Advances in protein structure prediction and design. Nat Rev Mol Cell Biol 2019, 20 (11), 681-697.

21. Alber, F.; Forster, F.; Korkin, D.; Topf, M.; Sali, A., Integrating diverse data for structure determination of macromolecular assemblies. Annu Rev Biochem 2008, 77, 443-77.

22. Saltzberg, D. J.; Viswanath, S.; Echeverria, I.; Chemmama, I. E.; Webb, B.; Sali, A., Using Integrative Modeling Platform to compute, validate, and archive a model of a protein complex structure. Protein Sci 2021, 30 (1), 250-261.

23. Rajabi, K.; Ashcroft, A. E.; Radford, S. E., Mass spectrometric methods to analyze the structural organization of macromolecular complexes. Methods 2015, 89, 13-21.

24. Seffernick, J. T.; Harvey, S. R.; Wysocki, V. H.; Lindert, S., Predicting Protein Complex Structure from Surface-Induced Dissociation Mass Spectrometry Data. ACS Cent Sci 2019, 5 (8), 1330-1341.

25. Aprahamian, M. L.; Chea, E. E.; Jones, L. M.; Lindert, S., Rosetta Protein Structure Prediction from Hydroxyl Radical Protein Footprinting Mass Spectrometry Data. Anal Chem 2018, 90 (12), 7721-7729.

26. Aprahamian, M. L.; Lindert, S., Utility of Covalent Labeling Mass Spectrometry Data in Protein Structure Prediction with Rosetta. Journal of Chemical Theory and Computation 2019, 15 (5), 3410-3424.

27. Biehn, S. E.; Lindert, S., Accurate protein structure prediction with hydroxyl radical protein footprinting data. Nat Commun 2021, 12 (1), 341.

28. Hall, Z.; Politis, A.; Robinson, Carol V., Structural Modeling of Heteromeric Protein Complexes from Disassembly Pathways and Ion Mobility-Mass Spectrometry. Structure 2012, 20 (9), 1596-1609.

29. Kahraman, A.; Herzog, F.; Leitner, A.; Rosenberger, G.; Aebersold, R.; Malmström, L., Cross-link guided molecular modeling with ROSETTA. PloS one 2013, 8 (9), e73411-e73411.

30. Tubb, M. R.; Silva, R. A.; Fang, J.; Tso, P.; Davidson, W. S., A three-dimensional homology model of lipid-free apolipoprotein A-IV using cross-linking and mass spectrometry. $J$ Biol Chem 2008, 283 (25), 17314-23.

31. Hauri, S.; Khakzad, H.; Happonen, L.; Teleman, J.; Malmström, J.; Malmström, L., Rapid determination of quaternary protein structures in complex biological samples. Nature Communications 2019, 10 (1), 192.

32. Saltzberg, D. J.; Broughton, H. B.; Pellarin, R.; Chalmers, M. J.; Espada, A.; Dodge, J. A.; Pascal, B. D.; Griffin, P. R.; Humblet, C.; Sali, A., A Residue-Resolved Bayesian Approach to Quantitative Interpretation of Hydrogen-Deuterium Exchange from Mass Spectrometry: Application to Characterizing Protein-Ligand Interactions. J Phys Chem B 2017, 121 (15), 34933501.

33. Zhang, M. M.; Beno, B. R.; Huang, R. Y.; Adhikari, J.; Deyanova, E. G.; Li, J.; Chen, G.; Gross, M. L., An Integrated Approach for Determining a Protein-Protein Binding Interface in Solution and an Evaluation of Hydrogen-Deuterium Exchange Kinetics for Adjudicating Candidate Docking Models. Anal Chem 2019, 91 (24), 15709-15717. 
34. Xie, B.; Sood, A.; Woods, R. J.; Sharp, J. S., Quantitative Protein Topography Measurements by High Resolution Hydroxyl Radical Protein Footprinting Enable Accurate Molecular Model Selection. Sci Rep 2017, 7 (1), 4552.

35. Politis, A.; Park, A. Y.; Hall, Z.; Ruotolo, B. T.; Robinson, C. V., Integrative Modelling Coupled with Ion Mobility Mass Spectrometry Reveals Structural Features of the Clamp Loader in Complex with Single-Stranded DNA Binding Protein. Journal of Molecular Biology 2013, 425 (23), 4790-4801.

36. Degiacomi, M. T., On the Effect of Sphere-Overlap on Super Coarse-Grained Models of Protein Assemblies. J Am Soc Mass Spectrom 2019, 30 (1), 113-117.

37. Eschweiler, J. D.; Frank, A. T.; Ruotolo, B. T., Coming to Grips with Ambiguity: Ion Mobility-Mass Spectrometry for Protein Quaternary Structure Assignment. J Am Soc Mass Spectrom 2017, 28 (10), 1991-2000.

38. Eschweiler, J. D.; Farrugia, M. A.; Dixit, S. M.; Hausinger, R. P.; Ruotolo, B. T., A Structural Model of the Urease Activation Complex Derived from Ion Mobility-Mass Spectrometry and Integrative Modeling. Structure 2018, 26 (4), 599-606.e3.

39. Wang, H.; Eschweiler, J.; Cui, W.; Zhang, H.; Frieden, C.; Ruotolo, B. T.; Gross, M. L., Native Mass Spectrometry, Ion Mobility, Electron-Capture Dissociation, and Modeling Provide Structural Information for Gas-Phase Apolipoprotein E Oligomers. J Am Soc Mass Spectrom 2019, 30 (5), 876-885.

40. Harvey, S. R.; Seffernick, J. T.; Quintyn, R. S.; Song, Y.; Ju, Y.; Yan, J.; Sahasrabuddhe, A. N.; Norris, A.; Zhou, M.; Behrman, E. J.; Lindert, S.; Wysocki, V. H., Relative interfacial cleavage energetics of protein complexes revealed by surface collisions. Proc Natl Acad Sci US A 2019, 116 (17), 8143-8148.

41. Graves, D. B., Transport properties of ions in gases by Edward A. Mason and Earl W. McDaniel, John Wiley and Sons, New York, 1988, $560+$ xvi pp. AIChE Journal 1989, 35 (4), 701-701.

42. Mason, E. A.; Schamp, H. W., Mobility of gaseous lons in weak electric fields. Annals of Physics 1958, 4 (3), 233-270.

43. Marklund, Erik G.; Degiacomi, Matteo T.; Robinson, Carol V.; Baldwin, Andrew J.; Benesch, Justin L. P., Collision Cross Sections for Structural Proteomics. Structure 2015, 23 (4), 791-799.

44. Mesleh, M. F.; Hunter, J. M.; Shvartsburg, A. A.; Schatz, G. C.; Jarrold, M. F., Structural Information from Ion Mobility Measurements: Effects of the Long-Range Potential. The Journal of Physical Chemistry 1996, 100 (40), 16082-16086.

45. Ewing, S. A.; Donor, M. T.; Wilson, J. W.; Prell, J. S., Collidoscope: An Improved Tool for Computing Collisional Cross-Sections with the Trajectory Method. J Am Soc Mass Spectrom 2017, 28 (4), 587-596.

46. Larriba, C.; Hogan, C. J., Free molecular collision cross section calculation methods for nanoparticles and complex ions with energy accommodation. Journal of Computational Physics 2013, 251, 344-363.

47. Shvartsburg, A. A.; Jarrold, M. F., An exact hard-spheres scattering model for the mobilities of polyatomic ions. Chemical Physics Letters 1996, 261 (1), 86-91.

48. Bleiholder, C.; Wyttenbach, T.; Bowers, M. T., A novel projection approximation algorithm for the fast and accurate computation of molecular collision cross sections (I). Method. International Journal of Mass Spectrometry 2011, 308 (1), 1-10. 
49. Bleiholder, C., A local collision probability approximation for predicting momentum transfer cross sections. Analyst 2015, 140 (20), 6804-13.

50. Mack, E., Average cross-sectional areas of molecules by gaseous diffusion methods. Journal of the American Chemical Society 1925, 47 (10), 2468-2482.

51. Webb, B.; Viswanath, S.; Bonomi, M.; Pellarin, R.; Greenberg, C. H.; Saltzberg, D.; Sali, A., Integrative structure modeling with the Integrative Modeling Platform. Protein Sci 2018, 27 (1), 245-258.

52. Leaver-Fay, A.; Tyka, M.; Lewis, S. M.; Lange, O. F.; Thompson, J.; Jacak, R.; Kaufman, K.; Renfrew, P. D.; Smith, C. A.; Sheffler, W.; Davis, I. W.; Cooper, S.; Treuille, A.; Mandell, D. J.; Richter, F.; Ban, Y.-E. A.; Fleishman, S. J.; Corn, J. E.; Kim, D. E.; Lyskov, S.; Berrondo, M.; Mentzer, S.; Popović, Z.; Havranek, J. J.; Karanicolas, J.; Das, R.; Meiler, J.; Kortemme, T.; Gray, J. J.; Kuhlman, B.; Baker, D.; Bradley, P., ROSETTA3: an objectoriented software suite for the simulation and design of macromolecules. Methods in enzymology 2011, 487, 545-574.

53. Leman, J. K.; Weitzner, B. D.; Lewis, S. M.; Adolf-Bryfogle, J.; Alam, N.; Alford, R. F.; Aprahamian, M.; Baker, D.; Barlow, K. A.; Barth, P.; Basanta, B.; Bender, B. J.; Blacklock, K.; Bonet, J.; Boyken, S. E.; Bradley, P.; Bystroff, C.; Conway, P.; Cooper, S.; Correia, B. E.; Coventry, B.; Das, R.; De Jong, R. M.; DiMaio, F.; Dsilva, L.; Dunbrack, R.; Ford, A. S.; Frenz, B.; Fu, D. Y.; Geniesse, C.; Goldschmidt, L.; Gowthaman, R.; Gray, J. J.; Gront, D.; Guffy, S.; Horowitz, S.; Huang, P. S.; Huber, T.; Jacobs, T. M.; Jeliazkov, J. R.; Johnson, D. K.; Kappel, K.; Karanicolas, J.; Khakzad, H.; Khar, K. R.; Khare, S. D.; Khatib, F.; Khramushin, A.; King, I. C.; Kleffner, R.; Koepnick, B.; Kortemme, T.; Kuenze, G.; Kuhlman, B.; Kuroda, D.; Labonte, J. W.; Lai, J. K.; Lapidoth, G.; Leaver-Fay, A.; Lindert, S.; Linsky, T.; London, N.; Lubin, J. H.; Lyskov, S.; Maguire, J.; Malmström, L.; Marcos, E.; Marcu, O.; Marze, N. A.; Meiler, J.; Moretti, R.; Mulligan, V. K.; Nerli, S.; Norn, C.; Ó'Conchúir, S.; Ollikainen, N.; Ovchinnikov, S.; Pacella, M. S.; Pan, X.; Park, H.; Pavlovicz, R. E.; Pethe, M.; Pierce, B. G.; Pilla, K. B.; Raveh, B.; Renfrew, P. D.; Burman, S. S. R.; Rubenstein, A.; Sauer, M. F.; Scheck, A.; Schief, W.; Schueler-Furman, O.; Sedan, Y.; Sevy, A. M.; Sgourakis, N. G.; Shi, L.; Siegel, J. B.; Silva, D. A.; Smith, S.; Song, Y.; Stein, A.; Szegedy, M.; Teets, F. D.; Thyme, S. B.; Wang, R. Y.; Watkins, A.; Zimmerman, L.; Bonneau, R., Macromolecular modeling and design in Rosetta: recent methods and frameworks. Nat Methods 2020, 17 (7), 665680.

54. Simons, K. T.; Kooperberg, C.; Huang, E.; Baker, D., Assembly of protein tertiary structures from fragments with similar local sequences using simulated annealing and Bayesian scoring functions. J Mol Biol 1997, 268 (1), 209-25.

55. Bradley, P.; Misura, K. M.; Baker, D., Toward high-resolution de novo structure prediction for small proteins. Science 2005, 309 (5742), 1868-71.

56. Raman, S.; Vernon, R.; Thompson, J.; Tyka, M.; Sadreyev, R.; Pei, J.; Kim, D.; Kellogg, E.; DiMaio, F.; Lange, O.; Kinch, L.; Sheffler, W.; Kim, B. H.; Das, R.; Grishin, N. V.; Baker, D., Structure prediction for CASP8 with all-atom refinement using Rosetta. Proteins 2009, 77 Suppl 9, 89-99.

57. Song, Y.; DiMaio, F.; Wang, R. Y.; Kim, D.; Miles, C.; Brunette, T.; Thompson, J.; Baker, D., High-resolution comparative modeling with RosettaCM. Structure 2013, 21 (10), 173542.

58. Alford, R. F.; Leaver-Fay, A.; Jeliazkov, J. R.; O’Meara, M. J.; DiMaio, F. P.; Park, H.; Shapovalov, M. V.; Renfrew, P. D.; Mulligan, V. K.; Kappel, K.; Labonte, J. W.; Pacella, M. 
S.; Bonneau, R.; Bradley, P.; Dunbrack, R. L.; Das, R.; Baker, D.; Kuhlman, B.; Kortemme, T.; Gray, J. J., The Rosetta All-Atom Energy Function for Macromolecular Modeling and Design. Journal of Chemical Theory and Computation 2017, 13 (6), 3031-3048.

59. Ismail, A. F.; Khulbe, K. C.; Matsuura, T., Fundamentals of Gas Permeation Through Membranes. In Gas Separation Membranes: Polymeric and Inorganic, Ismail, A. F.; Chandra Khulbe, K.; Matsuura, T., Eds. Springer International Publishing: Cham, 2015; pp 11-35.

60. Berman, H. M.; Battistuz, T.; Bhat, T. N.; Bluhm, W. F.; Bourne, P. E.; Burkhardt, K.; Feng, Z.; Gilliland, G. L.; Iype, L.; Jain, S.; Fagan, P.; Marvin, J.; Padilla, D.; Ravichandran, V.; Schneider, B.; Thanki, N.; Weissig, H.; Westbrook, J. D.; Zardecki, C., The Protein Data Bank. Acta Crystallographica Section D 2002, 58 (6 Part 1), 899-907.

61. Wang, G.; Dunbrack, R. L., Jr., PISCES: recent improvements to a PDB sequence culling server. Nucleic Acids Res 2005, 33 (Web Server issue), W94-8.

62. Stiving, A. Q.; Jones, B. J.; Ujma, J.; Giles, K.; Wysocki, V. H., Collision Cross Sections of Charge-Reduced Proteins and Protein Complexes: a Database for CCS Calibration. Analytical Chemistry 2020.

63. Allen, S. J.; Giles, K.; Gilbert, T.; Bush, M. F., Ion mobility mass spectrometry of peptide, protein, and protein complex ions using a radio-frequency confining drift cell. Analyst 2016, 141 (3), 884-891.

64. Faull, P. A.; Korkeila, K. E.; Kalapothakis, J. M.; Gray, A.; McCullough, B. J.; Barran, P. E., Gas-phase metalloprotein complexes interrogated by ion mobility-mass spectrometry. International Journal of Mass Spectrometry 2009, 283 (1), 140-148.

65. Hoaglund-Hyzer, C. S.; Counterman, A. E.; Clemmer, D. E., Anhydrous Protein Ions. Chemical Reviews 1999, 99 (10), 3037-3080.

66. Salbo, R.; Bush, M. F.; Naver, H.; Campuzano, I.; Robinson, C. V.; Pettersson, I.; Jørgensen, T. J. D.; Haselmann, K. F., Traveling-wave ion mobility mass spectrometry of protein complexes: accurate calibrated collision cross-sections of human insulin oligomers. Rapid Communications in Mass Spectrometry 2012, 26 (10), 1181-1193.

67. Kim, S. S.; Seffernick, J. T.; Lindert, S., Accurately Predicting Disordered Regions of Proteins Using Rosetta ResidueDisorder Application. J Phys Chem B 2018, 122 (14), 3920-3930.

68. Seffernick, J. T.; Ren, H.; Kim, S. S.; Lindert, S., Measuring Intrinsic Disorder and Tracking Conformational Transitions Using Rosetta ResidueDisorder. J Phys Chem B 2019, 123 (33), 7103-7112.

69. Gront, D.; Kulp, D. W.; Vernon, R. M.; Strauss, C. E.; Baker, D., Generalized fragment picking in Rosetta: design, protocols and applications. PLoS One 2011, 6 (8), e23294.

70. Bhardwaj, G.; Mulligan, V. K.; Bahl, C. D.; Gilmore, J. M.; Harvey, P. J.; Cheneval, O.; Buchko, G. W.; Pulavarti, S. V. S. R. K.; Kaas, Q.; Eletsky, A.; Huang, P.-S.; Johnsen, W. A.; Greisen, P., Jr.; Rocklin, G. J.; Song, Y.; Linsky, T. W.; Watkins, A.; Rettie, S. A.; Xu, X.; Carter, L. P.; Bonneau, R.; Olson, J. M.; Coutsias, E.; Correnti, C. E.; Szyperski, T.; Craik, D. J.; Baker, D., Accurate de novo design of hyperstable constrained peptides. Nature 2016, 538 (7625), 329-335.

71. Zhang, Y.; Skolnick, J., Scoring function for automated assessment of protein structure template quality. Proteins 2004, 57 (4), 702-10.

72. Zemla, A., LGA: A method for finding 3D similarities in protein structures. Nucleic acids research 2003, 31 (13), 3370-3374. 
73. Paizs, B., A divide-and-conquer approach to compute collision cross sections in the projection approximation method. International Journal of Mass Spectrometry 2015, 378, 360363.

74. Gray, J. J.; Moughon, S.; Wang, C.; Schueler-Furman, O.; Kuhlman, B.; Rohl, C. A.; Baker, D., Protein-Protein Docking with Simultaneous Optimization of Rigid-body Displacement and Side-chain Conformations. Journal of Molecular Biology 2003, 331 (1), 281-299.

75. Ohio Supercomputer Center. 1987. 
bioRxiv preprint doi: https://doi.org/10.1101/2021.05.27.445812; this version posted May 27, 2021. The copyright holder for this preprint (which was not certified by peer review) is the author/funder. All rights reserved. No reuse allowed without permission.

TOC Figure:

Ion Mobility Experiment

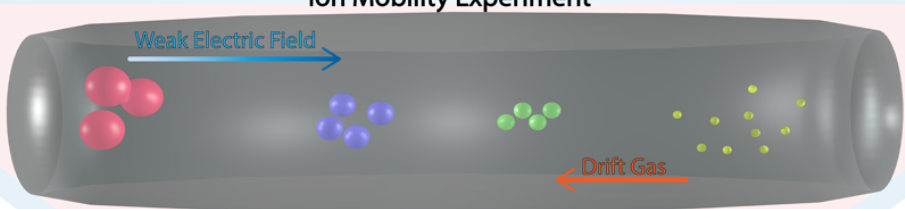

Projection Approximation

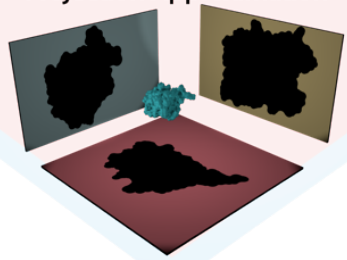

Protein Structure

Protein Structur

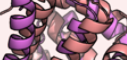

2us 